\title{
A Nonglycosylated 27 KDa Molecule as Common Antigen between Human Breast Cancer and Echinococcus granulosus Hydatid Cyst Wall
}

\author{
Seyedeh Maryam Sharafi' ${ }^{1}$, Raheleh Rafiei², Mahboubeh Hadipour ${ }^{2}$, Hedayat Shirzad ${ }^{3}$, \\ Hossein Khanahmad ${ }^{4}$, Hossein Yousofi Darani2* \\ ${ }^{1}$ Infectious Disease and Tropical Medicine Research Center, Isfahan University of Medical Sciences, Isfahan, Iran \\ ${ }^{2}$ Department of Medical Parasitology and Mycology, Isfahan University of Medical Sciences, Isfahan, Iran \\ ${ }^{3}$ Department of Immunology, Shahrekord University of Medical Sciences, Shahrekord, Iran \\ ${ }^{4}$ Department of Genetics, Isfahan University of Medical Sciences, Isfahan, Iran \\ Email: 'Yousofidarani@gmail.com
}

Received 1 March 2016; accepted 15 April 2016; published 19 April 2016

Copyright (C) 2016 by authors and Scientific Research Publishing Inc.

This work is licensed under the Creative Commons Attribution International License (CC BY).

http://creativecommons.org/licenses/by/4.0/

(c) (i) Open Access

\begin{abstract}
Background: Hydatid cyst, which has anti-cancer activities, is outwardly covered with the cyst wall. It is in close contact with the host tissues and its molecules presented to the immune system. In this work immunological reaction of the sera of breast cancer patients with the hydatid cyst wall antigens has been investigated. Method: For this purpose, sera of patients with breast cancer, hydatid cyst and normal human sera were collected and their reaction with hydatid cyst wall antigens was tested using western immunoblotting technique. Results: All sera of patients with breast cancer, hydatid cyst and also human normal sera reacted with a band in western immunoblotting. However, sera of patients with breast cancer showed reaction with a $27 \mathrm{KDa}$ band. Results of this work also revealed that this band was not glycosylated and may express only in some stages of breast cancer development. Conclusion: Sera of patients with breast cancer cross reacted with a nonglycosylated antigen of hydatid cyst wall. However, more work is recommended to find if this band involves in anticancer activity of the hydatid cyst.
\end{abstract}

\section{Keywords}

Breast Cancer, Cross Reaction, Hydatid Cyst

\footnotetext{
${ }^{*}$ Corresponding author.
}

How to cite this paper: Sharafi, S.M., Rafiei, R., Hadipour, M., Shirzad, H., Khanahmad, H. and Darani, H.Y. (2016) A Nonglycosylated 27 KDa Molecule as Common Antigen between Human Breast Cancer and Echinococcus granulosus Hydatid Cyst Wall. Advances in Breast Cancer Research, 5, 90-95. http://dx.doi.org/10.4236/abcr.2016.52010 


\section{Introduction}

Hydatid cyst is the larval stage of the tapeworm Echinococcus granulosus which is located in human and livestock viscera. Cysts act as space occupying lesion and put pressure on adjacent tissues. Clinical manifestations depend on the location of the cyst. Diagnosis of the disease relies on Para clinical methods including imaging and laboratory immunological techniques. The main therapy is surgery although albendazole is also used for treatment.

The cyst is outwardly covered with a carbohydrate rich material termed laminated layer [1]. Hydatid cyst, like most helminthes, polarizes the host immune response toward a Th2 type response [2]. However, this Th2 shift in hydatid cyst is not as marked as in other helminthes and a Th1 type response may also be detectable [3] [4]. Laminated layer is in close contact with host tissues and must be the major source of hydatid cyst molecules that immune system encounters.

Anticancer effect of hydatid cyst has been shown in cell culture experiments [5] [6] and also in animal model investigations [7] [8] or in human population [9]. However, the mechanism of this effect is still not clarified. Although this anti-cancer activity may be related to share antigens which exist between this parasite and cancers. In this context glycosilated antigens have been shown to exist both in hydatid cyst and some cancers [10]. Because cyst wall is in close contact with host tissues, in this work reaction of sera from patients with breast cancer and cyst wall antigens has been investigated.

\section{Materials and Methods}

Hydatid cyst antigens: In this descriptive study, sheep hydatid cysts were collected from khomainishahr slaughter house in Isfahan, Iran. The cysts were diagnosed according to their especial appearance in liver or lung and confirmed by observation of protoscolices in microscopic examination. Using sterile needle and syringe, hydatid cyst fluid was aspirated from the cyst and then collected in $50 \mathrm{ml}$ test tubes. One drop of the collected fluid was tested under microscope for presence of protoscolices (Figure 1). Hydatid fluid without protoscolices was discarded and ones with protoscolices were centrifuged at $2000 \mathrm{~g}$ for 2 minutes and the supernatant was collected as hydatid cyst fluid antigen. In order to make cyst wall antigen the cyst membrane existed from the cyst and then washed and sliced in phosphate buffer saline (PBS). The mixture was then sonicated, centrifuged and the supernatant was collected as cyst wall antigen.

Western immunoblotting: Sodium Dodecyl Sulfate Polyacrylamide Gel Electrophoresis (SDS-PAGE) was performed for both hydatid fluid and laminated layer antigen and the then transferred on nitrocellulose papers (NCP) as we published before for schistosomes [11]. The antigens in membrane were then coated with sera of patients with breast cancer or hydatid cyst. Normal human serum was used as control. Spare sera of patients with breast cancer were collected with informed consent from clinical laboratory of Saideoshohada hospital in Isfahan, Iran. Spare normal sera were collected from blood bank in Shahrekord, Iran.

Sodium metapenodate treatment of antigens on nitrocellulose paper: To inactivate carbohydrate epitopes, two NCP papers coated with the same antigen (case and control) were prepared. Then both of them washed with 0.1 $\mathrm{M}$ sodium acetate ( $\mathrm{pH}$ 4.5) for couple of minutes. The case paper was then incubated at $37^{\circ} \mathrm{C}$ for $1 \mathrm{~h}$ with 20 mM sodium m-periodate (Sigma) dissolved in $0.1 \mathrm{M}$ sodium acetate. The control paper was treated with $0.1 \mathrm{M}$ sodium acetate in the same conditions. After 2 washes with sodium acetate and one with PBS, the papers were

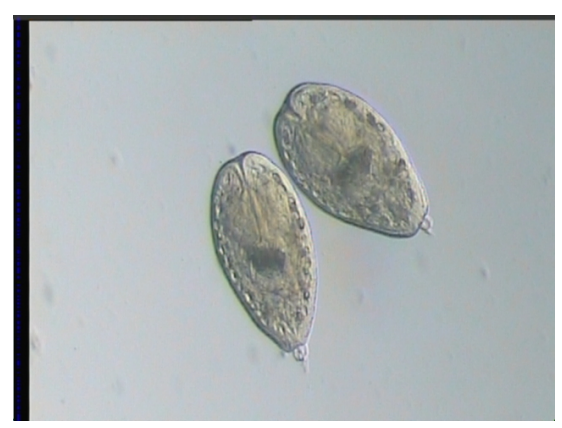

Figure 1. Protoscolices of hydatid cyst observed in hydatid cyst fluid under microscope with 100 times magnification. 
treated with $50 \mathrm{mM}$ sodium borohydride (Sigma) in PBS. The reaction was stopped after $30 \mathrm{~min}$ at room temperature by 5 washes with $10 \mathrm{mM}$ Tris/ $\mathrm{HCl}(\mathrm{pH}$ 8.0), $150 \mathrm{mM} \mathrm{NaCl}$, and $0.05 \%$ Tween-20. Subsequently, the routine western immunoblotting protocol was applied.

\section{Results}

In western immunoblotting cyst wall antigen and hydatid fluid antigens were probed with pooled sera of patients with breast cancer, hydatid cyst or normal human sera. All sera reacted with a band with molecular weight above $40 \mathrm{KDa}$ band both in cyst wall and hydatid cyst fluid. However, breast cancer sera reacted with a $27 \mathrm{KDa}$ band in laminated layer (Figure 2). To find if all individual sera of patients reacted with the $27 \mathrm{KD}$ band, the laminated layer was probed with sera of 10 individual patients with breast cancer. It was revealed that the reaction was not consistent in all patients' sera (Figure 3). To figure out if the reaction to cyst wall antigens related to glycan epitopes, cyst wall in NCP paper was treated with sodium metaperiodate and then probed with pooled sera of patients with breast cancer. The results showed that while the $27 \mathrm{KD}$ band was sodium metaperiodate resistant other bands disappeared following sodium metaperiodate treatment (Figure 4 \& Figure 5).

\section{Discussion}

Results of this work revealed that sera of patients with breast cancer cross reacted with a nonglycosylated $27 \mathrm{KD}$ band of hydatid cyst wall.

Immunological cross reaction between antibody against hydatid cyst and antibody against other parasites or antibody of patients with noninfectious diseases has been reported [12]. Common antigens among parasites have been reported, for example, it has been shown that Taenia crassiceps which is an animal infection and Taenias olium which is a human infection have some share antigens [13]. In another investigation immunological cross reaction among different helminthes antigens has also been reported [14]. Immunological cross reaction

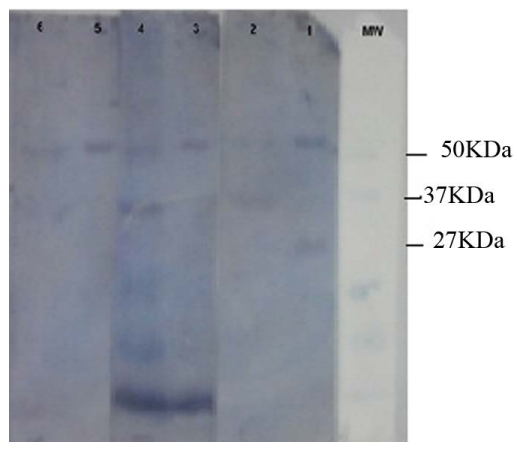

Figure 2. Western immunoblotting of cyst wall (lanes 1, 3 and 5) or hydatid cyst fluid (lanes 2, 4 and 6) probed with pooled sera of patients with breast cancer ( 1 and 2 ), hydatid cyst ( 3 and 4 ) or normal human sera (5 and 6). MW stands for molecular weight marker.

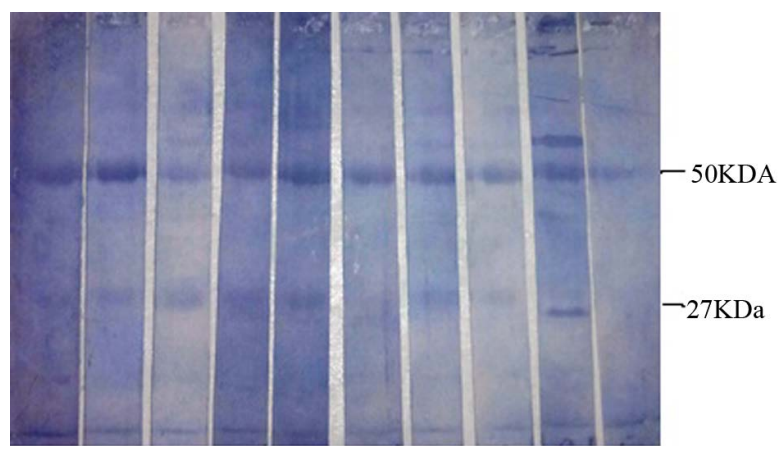

Figure 3. Western immunoblotting of the hydatid cyst wall antigens probed with sera of 10 individual patients with breast cancer. 


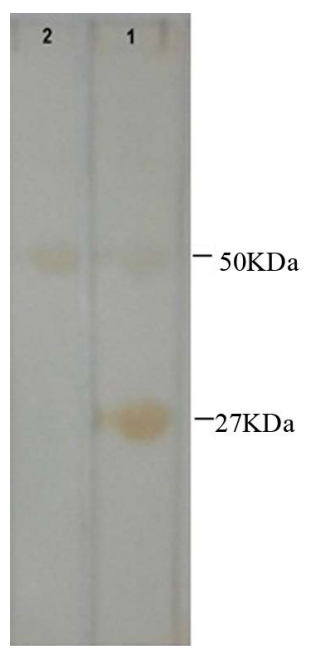

Figure 4. Western immunoblotting of the hydatid cyst wall antigens probed with the pooled sera of patients with breast cancer (1) or pooled normal human sera (2) before treatment with sodium metaperiodate.

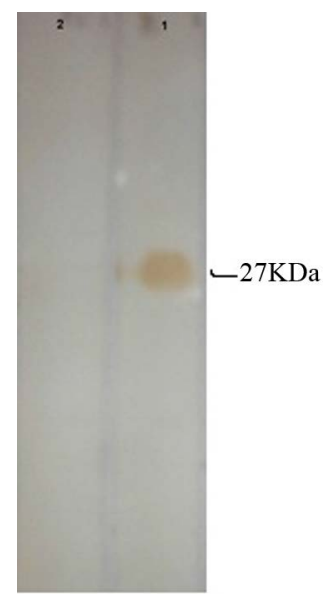

Figure 5. Western immunoblotting of the hydatid cyst wall antigens probed with the pooled sera of patients with breast cancer (1) or pooled normal human sera (2) after treatment with sodium metaperiodate.

between hydatid cyst antigens and other infections such as amoebic liver has also been reported [15].

Presence of share antigens between tumors and components of nervous system has been reported in the previous studies [16]. Also immunological cross reaction between Besnoitia besnoiti antigens and anti Neospora caninum has been shown [17].

It has been shown that much of the immunity to helminthes in mammalian hosts directed against glycan epitopes [18]. In hydatid cyst, it has also been shown that carbohydrates have an important contribution in host immune responses [19] [20]. So glycan epitopes may be responsible for majority of cross reactions that exist between hydatid cyst and other infectious and non-infectious agents. Although antibody response to helminthes is mostly directed against helminthes glycan epitopes, in our work sera of patients with breast cancer cross react with a peptide band of hydatid cyst. So it is interesting that a peptide molecule of the hydatid cyst laminated layer cross reacts with the sera of patients with breast cancer. It would be very worthwhile to sequence this peptide band in order to find if there is a similar molecule on the surface of cancer cells.

Results of the present investigation also revealed that reaction of sera of 10 patients with breast cancer reacted with the $27 \mathrm{KDa}$ band of hydatid cyst wall was not consistent among all individuals. Some sera showed strong 
reaction while others showed some weaker reaction. So it seems that this peptide antigen expresses in specific stage in breast cancer development. Therefore more investigations are needed to find if there is a correlation between expression of $27 \mathrm{KDa}$ band and the stage of the cancer.

Finally anti-cancer effect of hydatid cyst has been shown in different investigations [5]-[10] [21]-[25]. So it would be worthwhile to search whether this $27 \mathrm{KDa}$ molecule involves in anti-cancer activities of the hydatid cyst or not.

\section{Acknowledgements}

This work supported by grants from Isfahan University of Medical Sciences.

\section{Disclosure}

None of authors have conflict of interest.

\section{References}

[1] Díaz, A., Casaravilla, C., Irigoín, F., Lin, G., Previato, J.O. and Ferreira, F. (2011) Understanding the Laminated Layer of Larval Echinococcus I: Structure. Trends in Parasitology, 27, 204-213. http://dx.doi.org/10.1016/j.pt.2010.12.012

[2] Sher, A., Hieny, S., James, S. and Asofsky, R. (1982) Mechanisms of Protective Immunity against Schistosoma mansoni Infection in Mice Vaccinated with Irradiated Cercariae. II. Analysis of Immunity in Hosts Deficient in T Lymphocytes, B Lymphocytes, or Complement. The Journal of Immunology, 128, 1880-1884.

[3] Conchedda, M., Gabriele, F. and Bortoletti, G. (2004) Immunobiology of Cystic Echinococcosis. Parassitologia, 46, 375-380.

[4] Siracusano, A., Margutti, P., Delunardo, F., Profumo, E., Riganò, R., Buttari, B., Teggi, A. and Ortona, E. (2008) Molecular Cross-Talk in Host-Parasite Relationships: The Intriguing Immunomodulatory Role of Echinococcus Antigen B in Cystic Echinococcosis. International Journal for Parasitology, 38, 1371-1376. http://dx.doi.org/10.1016/j.ijpara.2008.06.003

[5] Hossein Yousofi, D. (2013) Effect of Different Hydatid Cyst Molecules on Hela and Vero Cell Lines Growth in Vitro. Journal of Immunodeficiency \& Disorders, 2, 1.

[6] Yousofi Darani, H., Soozangar, N., Khorami, S., Taji, F., Yousofi, M. and Shirzad, H. (2012) Hydatid Cyst Protoscolices Induce Cell Death in WEHI-164 Fibrosarcoma Cells and Inhibit the Proliferation of Baby Hamster Kidney Fibroblasts in Vitro. Journal of Parasitology Research, 2012, Article ID: 304183.

[7] Chookami, M.B., Sharafi, S.M., Sefiddashti, R.R., Jafari, R., Bahadoran, M., Pestechian, N. and Yousofi Darani, H. (2015) Effect of Two Hydatid Cyst Antigens on the Growth of Melanoma Cancer in $\mathrm{C}_{57} /$ Black Mice. Journal of Parasitic Diseases, 1-4. http://dx.doi.org/10.1007/s12639-015-0643-7

[8] Chookami, M., Sharafi, S., Sefiddashti, R., Bahadoran, M., Pestechian, N. and Yousofi Darani, H. (2014) Effect of Alive Protoscoleces of Hydatid Cyst on the Growth of Melanoma Cells in Mouse Model. Journal of Isfahan Medical School, 32.

[9] Akgül, H., Tez, M., Ünal, A.E., Keşkek, M., Sayek, İ. and Özçelik, T. (2003) Echinococcus against Cancer: Why Not? Cancer, 98, 1999-2000. http://dx.doi.org/10.1002/cncr.11752

[10] Darani, H.Y. and Yousefi, M. (2012) Parasites and Cancers: Parasite Antigens as Possible Targets for Cancer Immunotherapy. Future Oncology, 8, 1529-1535. http://dx.doi.org/10.2217/fon.12.155

[11] Darani, H. and Doenhoff, M. (2008) An Association between Schistosoma mansoni Worms and an Enzymatically-Active Protease/Peptidase in Mouse Blood. Parasitology, 135, 467-472. http://dx.doi.org/10.1017/S0031182007003988

[12] Daneshpour, S., Bahadoran, M., Yousefi, M., Mortazavi-Dehkordi, N., Mahmoudzadeh, M., Hejazi, S.H. and Yousofi Darani, H. (2014) Immunological cross Reaction between Cancer Cells and Hydatid Cyst. Journal of Shahrekord University of Medical Sciences, 16, 99-105.

[13] Espındola, N., De Gaspari, E., Nakamura, P. and Vaz, A. (2000) Cross-Reactivity of Anti-Taenia crassiceps Cysticerci Immune Antibodies with Taenia solium Antigens. Veterinary Parasitology, 89, 321-326. http://dx.doi.org/10.1016/S0304-4017(00)00208-9

[14] Ishida, M., Rubinsky-Elefant, G., Ferreira, A., Hoshino-Shimizu, S. and Vaz, A. (2003) Helminth Antigens (Taenia solium, Taenia crassiceps, Toxocara canis, Schistosoma mansoni and Echinococcus granulosus) and Cross-Reactivities in Human Infections and Immunized Animals. Acta Tropica, 89, 73-84. http://dx.doi.org/10.1016/j.actatropica.2003.09.005 
[15] Kanwar, J., Kaushik, S., Sawhney, I., Kamboj, M., Mehta, S. and Vinayak, V. (1992) Specific Antibodies in Serum of Patients with Hydatidosis Recognised by Immunoblotting. Journal of Medical Microbiology, 36, 46-51. http://dx.doi.org/10.1099/00222615-36-1-46

[16] Steinman, L. (2014) Conflicting Consequences of Immunity to Cancer versus Autoimmunity to Neurons: Insights from Paraneoplastic Disease. European Journal of Immunology, 44, 3201-3205. http://dx.doi.org/10.1002/eji.201445191

[17] García-Lunar, P., Moré, G., Campero, L., Ortega-Mora, L. and Álvarez-García, G. (2015) Anti-Neospora caninum and Anti-Sarcocystis spp. Specific Antibodies Cross-React with Besnoitia besnoiti and Influence the Serological Diagnosis of Bovine Besnoitiosis. Veterinary Parasitology, 214, 49-54. http://dx.doi.org/10.1016/j.vetpar.2015.09.011

[18] Dell, A., Haslam, S.M., Morris, H.R. and Khoo, K.-H. (1999) Immunogenic Glycoconjugates Implicated in Parasitic Nematode Diseases. Biochimica et Biophysica Acta, 1455, 353-362. http://dx.doi.org/10.1016/S0925-4439(99)00064-2

[19] Sterla, S., Ljungström, I. and Nieto, A. (1997) Modified ELISA for Hydatid Serodiagnosis: The Potential of Periodate Treatment and Phosphorylcholine Inhibition. Serodiagnosis and Immunotherapy in Infectious Disease, 8, 145-148. http://dx.doi.org/10.1016/S0888-0786(96)01069-4

[20] Sterla, S., Sato, H. and Nieto, A. (1999) Echinococcus granulosus Human Infection Stimulates Low Avidity Anticarbohydrate IgG2 and High Avidity Antipeptide IgG4 Antibodies. Parasite Immunology, 21, 27-34. http://dx.doi.org/10.1046/j.1365-3024.1999.00197.x

[21] Arefkhah, N., Shirzad, H., Mosavi, F., Taghipor, S., Daneshpor, S. and Yousofi, D.H. (2013) Effects of Hydatid Cyst Antigens on Hella Cells in Vitro. Journal of Shahrekord University of Medical Sciences, 15, 65-71.

[22] Dorosti, Z., Yousefi, M., Sharafi, S.M. and Darani, H.Y. (2014) Mutual Action of Anticancer and Antiparasitic Drugs: Are There Any Shared Targets? Future Oncology, 10, 2529-2539. http://dx.doi.org/10.2217/fon.14.65

[23] Darani, H.Y., Yousefi, M., Safari, M. and Jafari, R. (2014) Parasites and Immunotherapy: With or against? Journal of Parasitic Diseases, 1-10.

[24] Shirzad, H., Khorami, S., Soozangar, N., Yousefi, M. and Darani, H.Y. (2012) Toxoplasma gondii but Not Leishmania major or Trichomonas vaginalis Decreases Cell Proliferation and Increases Cell Death on Fibrosarcoma Cancer Cells in Culture Medium. World Journal of Vaccines, 2, 105-108. http://dx.doi.org/10.4236/wjv.2012.22014

[25] Darani, H.Y., Shirzad, H., Mansoori, F., Zabardast, N. and Mahmoodzadeh, M. (2009) Effects of Toxoplasma gondii and Toxocara canis antigens on WEHI-164 Fibrosarcoma Growth in a Mouse Model. The Korean Journal of Parasitology, 47, 175-177. http://dx.doi.org/10.3347/kjp.2009.47.2.175

\section{List of Abbreviations}

NCP: Nitrocellulose paper. PBS: Phosphate buffer saline.

SDS PAGE: Sodium Dodecyl Sulfate Polyacrylamide Gel Electrophoresis. 